\title{
Mejoramiento del clima institucional a través de estrategias de gestión en los docentes de la IE José Lorenzo Cornejo Acosta de Arequipa.
}

Improvement of the institutional climate through management strategies in the teachers of the IE José Lorenzo Cornejo Acosta de Arequipa.

Ivette Shirley Pariente Arenas

Instituto de Educación Superior Pedagógico Público. Arequipa Perú.

\section{INFORMACIÓN}

\section{Historia del Artículo}

Recepción: 27/12/2019

Revisión: $15 / 02 / 2020$

Aceptación: 20/05/2020

\section{Palabras Clave}

Clima institucional, estrategias de gestión, programa.

\section{Key Words}

Institutional climate, management strategies, program.

\section{DOI}

https://doi.org/10.35286/veritas. v21i2.273

\begin{abstract}
RESUMEN
La investigación tuvo como objetivo la aplicación de un programa de estrategias de gestión sustentado en las teorías de las relaciones humanas de Elton Mayo y de la inteligencia emocional de Daniel Goleman, para mejorar el clima institucional entre el personal docente de la IE José Lorenzo Acosta, de la ciudad de Arequipa. Por lo que se formuló el siguiente problema ¿Qué características presenta el clima institucional de entre los docentes de la IE José Lorenzo Acosta de la ciudad de Arequipa? En el estudio diagnóstico que se realizó en una muestra de 46 docentes, se utilizó una encuesta dirigida al personal de la Institución Educativa determinándose la falta de compromiso, el no respeto a las normas de convivencia, intolerancia, falta de comunicación y poca empatía entre sus miembros. La contrastación de las hipótesis planteadas permite determinar que se redujeron los problemas de clima institucional existente, ya que las actitudes desfavorables disminuyeron notablemente.
\end{abstract}

\begin{abstract}
The main goal of the research was to apply a program of management strategies based on Elton Mayo's theories of human relations and the emotional intelligence of Daniel Goleman, to improve the working environment in the teaching staff of the IE José Lorenzo Acosta, from the city of Arequipa. In that way we have proposed the following problem: What are the characteristics of the institutional climate among teachers in José Lorenzo Acosta School from Arequipa city? In the diagnostic study that was carried out in a sample of 46 teachers, a survey was used directed to the staff of the Educational Institution determining the lack of commitment, the lack of respect for the rules of coexistence, intolerance, lack of communication and little empathy among their members. The comparison of the hypotheses allows to determine that the problems of the existing institutional climate were reduced, since the unfavorable attitudes diminished remarkably.
\end{abstract}

\section{INTRODUCCIÓN}

El clima institucional es el ambiente generado en una institución educativa a partir de las vivencias cotidianas de los miembros de la institución. Este ambiente incluye aspectos tales como el trato entre los miembros, relaciones interpersonales, comunicación y el estilo de gestión. Por lo tanto es importante, ya que si es así el desarrollo del trabajo será muy favorable tanto en los procesos de enseñanza aprendizaje como en las relaciones interpersonales, y por ende, en el logro de los objetivos institucionales.

Méndez (2006) define "el clima institucional como el resultado de la forma como las personas establecen procesos de interacción social y en donde dichos procesos están influenciados por un sistema de valores, actitudes y creencias, así como también en un ambiente interno" Un clima institucional favorable o adecuado es fundamental para un funcionamiento eficiente de la institución educativa, donde los valores, actitudes y creencias, así como un ambiente interno así como crear condiciones de convivencia armoniosa.

El problema de investigación en la institución Educativa José Lorenzo Acosta se refiere al deterioro del clima institucional, entre su personal docente por lo que se evidencia la falta de compromiso, sin respeto por las normas de convivencia mostrando inadecuada relaciones personales, falta de tolerancia, falta de comunicación y poca empatía entre sus miembros.

Algunos trabajos de investigación tales como los realizados por Zevallos Luque y Rosario Jeanette (2007) denominado Diseño de Estrategia de motivación para mejorar el desarrollo institucional en la institución Educativa 40002 "Al aire Libre" o el de Duman Reyna Lujan intitulado La calidad educativa y el clima institucional, reconocen la importancia de promover el clima institucional que facilite el cambio, es la de flexibilizar la institución, para responder a los continuos, complejos y relevantes cambios que se producen en el contexto social y educativo, que se logrará desde la perspectiva de que las instituciones deben "desaprender y volver a aprender". 


\section{MÉTODOS Y MATERIALES}

El diseño de esta investigación está enmarcado en el paradigma cuantitativo. El método y diseño es de carácter cuasi experimental. El motivo de esta investigación utiliza estas características debido a la necesidad de probar un caso de forma experimental. Este diseño como Sierra (1996) menciona se basa en el supuesto de que la variación de una a otra medida se debe al influjo de la variable experimental, lo que quedará directamente evidenciado en el análisis del grupo experimental.

En situaciones donde por algún motivo se cuenta con un número reducido de sujetos para el experimento, se puede llevar a cabo un diseño con tratamientos múltiples y un solo grupo. No hay asignación al azar puesto que se tiene a un único grupo. La equivalencia se obtiene puesto que no hay nada más similar a un grupo que éste mismo. El grupo hace las veces de "grupos experimentales" y de control" (Hernández 1994). La investigación cuasi-experimental en la que existe un hipótesis para contrastar, pero no hay aleatorización de los sujetos a los grupos de tratamiento y control, es decir no existe grupo de control

En esta investigación participaron el personal de la institución educativa José Lorenzo Cornejo Acosta que se distribuye de la siguiente manera: personal docente 46 , personal administrativo 5, estudiantes 889 , en total 940 personas forman la comunidad educativa. La muestra viene a ser 22 del nivel primario y del nivel secundario 24 , siendo el total 46 docentes que participan directamente en la investigación. El diseño muestral fue de carácter no probabilístico y por conveniencia.

Una vez revisada la literatura, es necesario indicar que la propuesta de intervención que se presenta en este documento se centra en la aplicación de un programa constituido por estrategias de gestión para mejorar el clima institucional denominado FOMEPRACDINA (fortaleciendo, mejorando, practicando y dinamizando las relaciones interpersonales).

La propuesta se establece a partir del diseño de estrategias compuestas por la ejecución de talleres grupales que posibiliten el desarrollo de un clima institucional positivo, teniendo en cuenta los siguientes factores: las relaciones interpersonales, que se mejora con el buen trato; la inteligencia emocional y los principios de gestión referidos a la comunicación y transparencia. Se realizaron 11 talleres organizados en seis temáticas: fortaleciendo la confianza y el compromiso, mejorando el ambiente de trabajo, practicando las normas de convivencia, mejorando la empatía entre sus miembros, dinamizando la comunicación entre agentes educativos, y finalmente actividades de integración.

Dado que la muestra está constituida por población docente, se obtuvo la autorización de la dirección de la institución educativa para el desarrollo del trabajo y la colaboración del profesorado, siendo informados por escrito previamente del objetivo de la investigación.

\section{RESULTADOS}

El clima está conformado por las percepciones de las variables de comportamiento, estructura y procesos. Según Chruden y Sherman (1982), toda organización posee su propia y exclusiva personalidad o clima que la diferencia de otros. Dichos autores sostienen que la gerencia debe prestar mucha atención a este aspecto, ya que entender el clima de una organización conlleva al logro de los objetivos propuestos.

El clima en organizaciones educativas para autores, como Fernández y Asensio (1989), es el conjunto de características psico-sociales de un centro educativo, determinado por todos aquellos factores o elementos estructurales, personales y funcionales de la institución que contienen un peculiar estilo, condicionantes, a su vez, de sus productos educativos.

El clima institucional del personal docente de la Institución Educativa José Lorenzo Cornejo Acosta, estaba deteriorado lo que se evidenció en los resultados del pre test, que mostraba la falta de compromiso, el no respeto a las normas de convivencia, intolerancia, falta de comunicación y poca empatía entre sus miembros.

Se elaboró y aplicó la propuesta, lo que dio como resultado, al comparar los resultados del pre y post test que se redujeron los problemas de clima institucional existente, ya que las actitudes desfavorables disminuyeron notablemente, tal como se evidencia en el post test, ya que permitió mejorar la: confianza y compromiso, el ambiente de trabajo, el cumplimiento de normas de convivencia y las comunicaciones por lo tanto mejoró el clima institucional. Con ello se cumplieron los objetivos y quedó validada la hipótesis de la presente investigación.

\section{CONCLUSIONES}

Se puede concluir afirmando que, según las aportaciones de este trabajo, es posible modificar la forma de pensar y actuar de los docentes con respecto al clima institucional, incidiendo en su mejora y conservación. No obstante, para que esto sea posible, es necesario que exista una participación activa por parte del profesorado, con un programa bien organizado.

Una vez revisada la hipótesis y los resultados emanados en esta investigación se puede obtener alguna recomendación importante para mejorar propuestas de intervención enfocadas en el clima institucional. Por ejemplo: involucrar a los docentes en actividades de varios días en entornos distintos a la institución educativa donde pudieran conocer y llevar a cabo actividades propias de estos lugares. Una posible futura línea de investigación podría ser la realización de un programa con actividades de este tipo tanto con docentes como con personal administrativo.

\section{DISCUSIÓN}

En cuanto a los resultados sobre la comunicación con los directivos de la institución educativa, un $42 \%$ manifestaba que se da rara vez o nunca, siendo la comunicación indispensable para las buenas relaciones entre las personas y sobre todo en una I.E. para generar un clima institucional positivo, sobretodo porque las relaciones interpersonales están basadas en la comunicación y es valorada como herramienta fundamental de la interacción social, debiendo ser veraz y oportuna, de ida y retorno y darse con actitud de diálogo y de escucha.

Respecto a la libertad para expresarse el $44 \%$ de los docentes manifestaron que no la cabe mencionar que la libertad de expresión que se evidencia en la institución sea ido generando a raíz de que se les de la confianza necesaria 
y sin represalias, situación que en la institución educativa se irá incrementando generando confianza y seguridad para hacerlo.

Básicamente en los dos niveles en los que debe aplicarse la libertad de expresión: el individual (que se refiere a la libertad para que cada persona exprese libremente sus ideas y pensamientos) y el colectivo (que señala el intercambio de información para la participación de quienes integran la sociedad).

En síntesis, debemos señalar, que el clima institucional tiene un carácter multidimensional, que incluye numerosas variables: buenas relaciones, participación, cooperación, tolerancia, flexibilidad, estilo de liderazgo y características del contexto que rodea a la institución educativa, por lo tanto, son también múltiples las actividades que deben desarrollarse para desarrollar y fortalecer el clima en la institución,

\section{REFERENCIAS BIBLIOGRÁFICAS}

1. Chruden, H. \&Sherman, A. (1982). Administración de personal. México: Editorial Continente

2. Fernandez, M. \& Asensio, (1989a). Concepto de clima institucional. APUNTES DE EDUCACIÓN, DIRECCIÓN Y ADMINISTRACIÓN

3. Hernández, S. R., Fernández, C. C., \& Baptista, L.P. (2014). Metodología de la investigación (Vol. 3): México: McGraw-Hill.

4. Hernández S. y otros (1994), Metodología de la Investigación, MÉXICO, MC GRAW HILL

5. Méndez, C. (2006). Cultura organizacional de éxito: London - dorling kindersley limited

6. Sierra, R. (1996). Tesis Doctorales y Trabajos de Investigación Científica. MADRID: EDITORIAL PARANINFO. 